\title{
Regions Development and Convection Motion in Horizontally Vibrated Granular System
}

\author{
Abdul Rehman ${ }^{a, \dagger}$, Sana Shoukat ${ }^{b, *}$, Ping Wu ${ }^{a}$, And Li Wang ${ }^{c}$ \\ ${ }^{a}$ School of Mathematics and Physics, University of Science \& Technology Beijing, Beijing, 100083, China \\ ${ }^{b}$ College of Biological Sciences and Biotechnology, Beijing Forestry University, Beijing, 100083, China \\ ${ }^{c}$ School of Energy and Environmental Engineering, University of Science \& Technology Beijing, \\ Beijing, 100083, China \\ (Received September 5, 2017) \\ This research examined a horizontally vibrated monolayer of granular particles system at 5, 10, and $15 \mathrm{~Hz}$ \\ frequencies and dimensionless acceleration $\Gamma$ varied from 0.8 to 1.4. Different modes of convection such as non- \\ convection mode, two rolls convection mode, one roll clockwise convection mode, one roll anticlockwise convection \\ mode and different density regions such as 3D state, gassy, empty, cluster and solid regions were observed in the \\ system. Empty region size affects the densities of all regions and no prominent effect of cluster region was observed \\ on convection modes. Kinetic energy ratio of gassy and solid regions $\mu=k_{g} / k_{s}$ described the modes of convection. \\ When $\mu=0.02$ non-convection mode, $\mu=5.36$ two rolls convection mode, $\mu=6.23$ 3D state mode and one roll \\ convection mode, $\mu=6.85$ one roll clockwise convection mode, and $\mu=8.76$ one roll anticlockwise convection \\ mode were observed in the system.
}

DOI: 10.12693/APhysPolA.134.484

PACS/topics: pattern selection (fluid dynamics), convection, rheology of complex fluids

\section{Introduction}

Granular materials are assemblies of dissipative particles. Numerous investigations on collective behaviors and non-equilibrium phase transitions have been witness [1$6]$. The study does not have an essential physical curiosity, but it is significant for countless industries [7-10]. Various collective behaviors and non-equilibrium phase transitions demonstrate a prominent resemblance with solid or fluids phenomena [11-14]. Granular materials divulge a number of exhilarating phenomena, such as selforganized convection motion and various types of segregation [15-17]. Horizontally shaken systems possess complex patterns of convection [18-22]; it is also a stimulating research issue [23-29]. The transition between solid and fluid states was also witnessed in 2D experiments [30] and simulations [21, 30-32]. Granular materials remain in a gaseous state, the most peculiar feature that differentiates them from molecular gases is their tendency to form highly concentrated and diluted regions [8, 33]. Five different states namely: unstable-gas, homogeneous gas, one-clustering, two-clustering, and granular oscillation states have been observed [7].

We discussed earlier, four different regions: solid, cluster, gassy and empty regions and the effect of moment of inertia on energy dissipation and convection motion [34]. Densities and kinetic energies of these regions influence the convection motion. More efforts are required for un-

\footnotetext{
*corresponding author; e-mail: sanashoukat@ymail.com

${ }^{\dagger}$ corresponding author; e-mail: ideal166@hotmail.com
}

derstanding the mechanics behind the development of regions and the effect of regions on convection motion. This investigation will discuss the development of different regions and different modes of convection as well as the regions affect the modes of convection.

\section{Experimental setup}

Our experimental system was comprised of a translucent smooth square container of magnitude $L_{X} \times L_{Y} \times$ $L_{Z}=25 \times 25 \times 18 \mathrm{~cm}^{3}$, filled with $400 \mathrm{~g}$ of granular particles which was then fixed flat on a vibrator. The density and the diameter of particles were $\rho=1.05 \mathrm{~g} / \mathrm{cm}^{3}$ and $d=8.00 \pm 0.02 \mathrm{~mm}$, respectively. A function generator and an electromagnetic vibrator provided sinusoidal vibration in horizontal direction. Experimental setup sketch is shown in Fig. 1. Three different frequencies 5,10 , and $15 \mathrm{~Hz}$ were investigated while the dimensionless acceleration $\Gamma=A \omega^{2} / g$ varied from 0.8 to 1.4. A portable accelerometer UNI-T (UT311) directly attached on the vibrator to measure the magnitude of acceleration $a=A(2 \pi f)^{2}$. Revealer $2 \mathrm{~F} 01$ video system

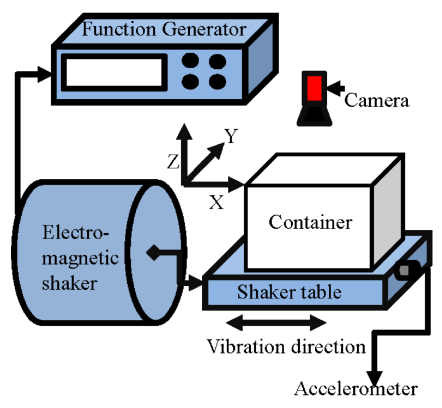

Fig. 1. Sketch of experimental setup. 
was used to observe the movement of particles. Images taken from the camera were processed through MATLAB image processing tool to count the number of particles, mass, and density of each region. Particle image velocimetry (PIV) was used to calculate the speed of each region and by using these velocities and densities of the regions we have calculated the kinetic energy of each region as shown in Table I.

TABLE I

Experimental data. Modes: A - no convection, B - two roll convection, $\mathrm{C}$ - one roll anticlockwise convection, $\mathrm{D}$ - one roll clockwise convection, $\mathrm{E}-3 \mathrm{D}$ state.

\begin{tabular}{l|c|c|c|c|c}
\hline \hline Mode & A & B & C & D & E \\
\hline \multicolumn{6}{c}{ solid region } \\
\hline $\bar{m}_{s}[\mathrm{~g}]$ & 375.18 & 352.56 & 365.54 & 355.74 & 367.34 \\
$\bar{v}_{s}[\mathrm{~cm} / \mathrm{s}]$ & 0.46 & 0.68 & 0.82 & 1.01 & 1.03 \\
$\bar{k}_{s}[\mathrm{~g} \mathrm{~cm} / \mathrm{s}]$ & 42.48 & 80.31 & 125.25 & 197.11 & 202.35 \\
$\bar{\rho}_{s}\left[\mathrm{~g} / \mathrm{cm}^{3}\right]$ & 0.91 & 1.04 & 1.06 & 1.07 & 1.06 \\
\hline \multicolumn{6}{c}{ cluster region } \\
\hline $\bar{m}_{c}[\mathrm{~g}]$ & 20.09 & 30.63 & 15.56 & 25.32 & 20.74 \\
$\bar{v}_{c}[\mathrm{~cm} / \mathrm{s}]$ & 0.21 & 0.39 & 0.25 & 0.21 & 0.33 \\
$\bar{k}_{c}[\mathrm{~g} \mathrm{~cm} / \mathrm{s}]$ & 4.11 & 9.08 & 7.47 & 4.87 & 9.96 \\
$\bar{\rho}_{c}\left[\mathrm{~g} / \mathrm{cm}^{3}\right]$ & 0.20 & 0.21 & 0.17 & 0.21 & 0.23 \\
\hline \multicolumn{6}{c}{ gassy region } \\
$\bar{m}_{g}[\mathrm{~g}]$ & 6.53 & 18.62 & 20.70 & 20.74 & 13.72 \\
$\bar{v}_{g}[\mathrm{~cm} / \mathrm{s}]$ & 0.17 & 3.40 & 9.45 & 9.23 & 13.63 \\
$\bar{k}_{g}[\mathrm{~g} \mathrm{~cm} / \mathrm{s}]$ & 0.82 & 430.49 & 1097.51 & 1351.42 & 1262.42 \\
$\bar{\rho}_{g}\left[\mathrm{~g} / \mathrm{cm}^{3}\right]$ & 0.09 & 0.12 & 0.19 & 0.27 & 0.15
\end{tabular}

\section{Results and discussion}

When the system was vibrated horizontally with 5,10 , and $15 \mathrm{~Hz}$ fixed frequencies and dimensionless acceleration $\Gamma$ varying from 0.8 to 1.4 , four different density regions and different modes of convection were observed. We previously expressed the names of the regions as solid, cluster, gassy and empty region in Ref. [33]. In this work we have calculated the densities of each region to make it clearer. When particles are very close to each other with density $\rho_{s}=0.7-1.1 \mathrm{~g} / \mathrm{cm}^{3}$ it is defined as the solid region. Where particles are in groups with density $\rho_{c}=0.4-0.7 \mathrm{~g} / \mathrm{cm}^{3}$ it is defined as the cluster region. When there are large gaps between the particles with density $\rho_{g}=0.2-0.4 \mathrm{~g} / \mathrm{cm}^{3}$ it is defined as the gassy region. When no particles are present with density $\rho_{e}=0 \mathrm{~g} / \mathrm{cm}^{3}$ it is defined as the empty region. Different modes of convection were also observed in the system such as non-convection mode, two rolls convection mode, one roll clockwise convection mode and one roll anti- clockwise convection mode. Solid, gassy and empty regions showed the most dominating effects on the modes of convection. Where $\bar{m}_{s}, \bar{v}_{s}, \bar{k}_{s}$, and $\bar{\rho}_{s}$ represent average mass, velocity, kinetic energy, and density of solid region, respectively. $\bar{m}_{c}, \bar{v}_{c}, \bar{k}_{c}$, and $\bar{\rho}_{c}$ represent average mass, velocity, kinetic energy and density of region, respectively. $\bar{m}_{g}, \bar{v}_{g}, \bar{k}_{g}$, and $\bar{\rho}_{g}$ represent average mass, velocity, kinetic energy, and density of gassy region, respectively. Colors in Figs. 2-6 differentiate the regions. Red color represents gassy region, blue color represents empty region, green color represent cluster region and uncolored part is represented by the solid region.

\subsection{Regions development in non convection mode}

When the system was vibrated with 5,10 , and $15 \mathrm{~Hz}$ frequency and dimensionless acceleration $\Gamma$ was 0.8 , no convection motion was witnessed, but different regions appeared in the system. At $5 \mathrm{~Hz}$ two gassy regions appeared in corner 1 and corner 3 and dense solid regions were observed in corner 2 and corner 4 of the container as shown in Fig. 2a. At $10 \mathrm{~Hz}$ a large cluster region appeared on wall 2 of container as shown in Fig. $2 \mathrm{~b}$ and at $15 \mathrm{~Hz}$ a large empty region appeared on corner 1 of the container as shown in Fig. 2c, other parts of system were covered by solid region.

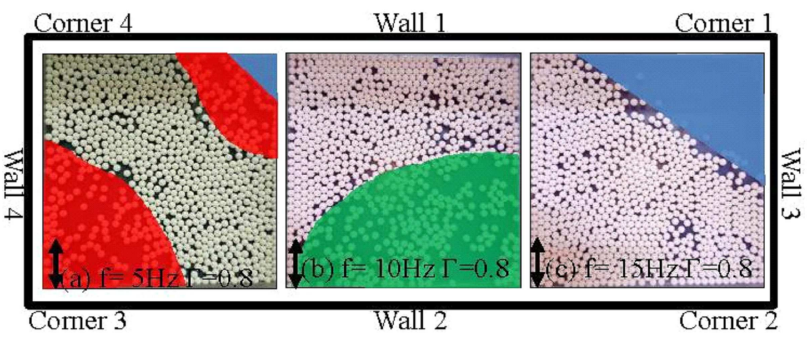

Fig. 2. Region appeared in non-convection mode, red color represents gassy region, blue color represents empty region, green color represent cluster region and uncolored part is solid region. Bi-direction arrow represents the direction of vibration.

\subsection{Regions development in two convection rolls mode}

Two convection rolls mode was observed at $10 \mathrm{~Hz}$ and $15 \mathrm{~Hz}$ frequency and dimensionless acceleration $\Gamma$ was 1.0. At $10 \mathrm{~Hz}$ large cluster region appeared in corner 2 and two empty regions appeared in corners 1 and 3 of the container as shown in Fig. 3a. At $15 \mathrm{~Hz}$ small empty regions appeared in corner 1 and large empty region appeared on corner 3 and after the empty region a gassy region appeared on wall 2 of the container as shown in Fig. 3b. Small red arrow in Fig. 3 shows the direction of motion of particles.

\subsection{Regions development in one roll clockwise convection mode}

One roll clockwise convection mode was observed at $(f=10 \mathrm{~Hz}, \Gamma=1.2)$ and $(f=15 \mathrm{~Hz}, \Gamma=1.2-1.4)$. At $f=10 \mathrm{~Hz}, \Gamma=1.2$ two small empty regions in corners 1 and 3 and a big gassy region at the center of wall 2 were in the system as shown in Fig. 4a. When $f=15 \mathrm{~Hz}$, $\Gamma=1.2$ a large gassy region appeared on wall 4 as shown in Fig. 4b. When $f=15 \mathrm{~Hz}, \Gamma=1.4$ three empty regions appeared in corners 1,2,3 and a gassy region appeared after the empty region in corner 3 of the container as shown in Fig. 4c. 


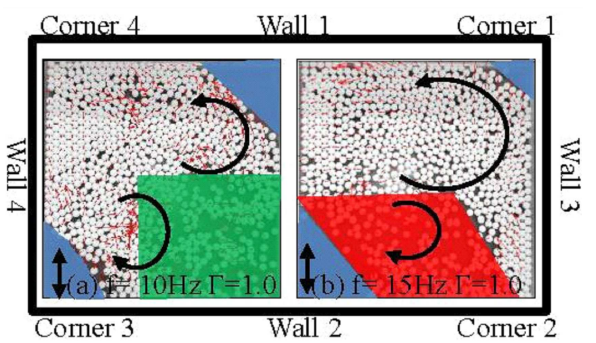

Fig. 3. Region appearing in two convection roll mode, red color represents gassy region, blue color represents empty region, green color represent cluster region and uncolored part represents the solid region. Bi-direction arrow represents the direction of vibration. Small red arrow and black circular arrow represent the direction of convection rolls.

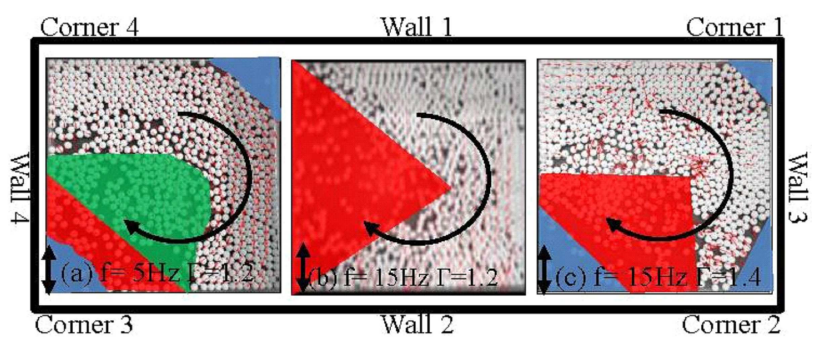

Fig. 4. PIV results: region appearing in one roll clockwise convection mode, red color represents gassy region, blue color represents empty region, green color represent cluster region and uncolored part is solid region. Bi-direction arrow represents the direction of vibration. Small red arrow and black circular arrow represent the direction of convection.

\subsection{Regions development in one roll anticlockwise convection mode}

One roll anticlockwise convection roll mode was observed at $(f=5 \mathrm{~Hz}, \Gamma=1.0$ and 1.4$)$ and $(f=10 \mathrm{~Hz}$, $\Gamma=1.2-1.4)$. When the system was vibrated with $f=5 \mathrm{~Hz}, \Gamma=1.0$ empty regions appeared in corner 1 and corner 3 and a gassy region appeared in corner 3 after the empty region as shown in Fig. 5a. At $f=5 \mathrm{~Hz}$, $\Gamma=1.0$ three empty regions appeared in corner $1,2,3$ and a gassy region appeared at the center of wall 2 as shown in Fig. 5 b. At $f=10 \mathrm{~Hz}, \Gamma=1.4$ a small empty region appeared in corner 1 along with a large empty and a large cluster region on wall 2 of container as shown in Fig. 5d.

\subsection{Regions development in 3D state mode}

When the system was vibrated with 5,10 , and $15 \mathrm{~Hz}$ frequency and dimensionless acceleration $\Gamma$ was $1.4,3 \mathrm{D}$ state was always observed in corner 4 of the container. At $5 \mathrm{~Hz}$ three small empty regions were observed in corners $1,2,3$ and a big gassy region at the center of wall 2 as shown in Fig. 6a. At $10 \mathrm{~Hz}$ a small empty regions was observed in corner 1 and a large empty region as well as a large cluster region were observed on wall 2 as shown in Fig. 6b. At $15 \mathrm{~Hz}$ empty regions appeared in corners 1,

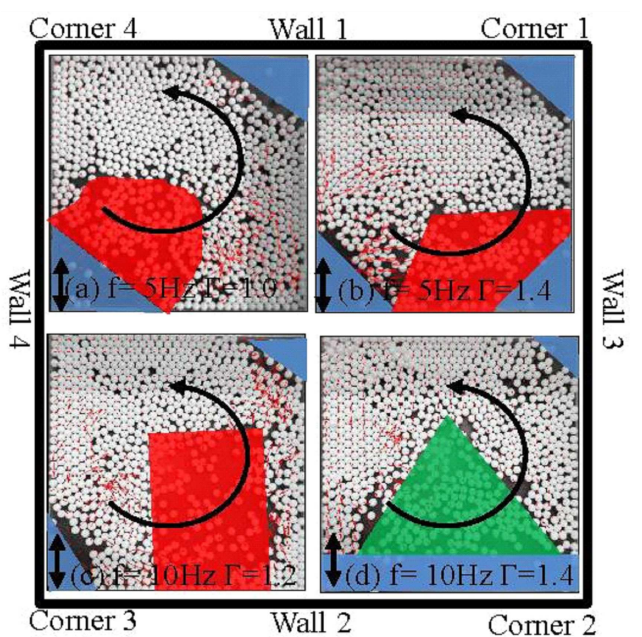

Fig. 5. PIV results: region appeared in one roll anticlockwise convection mode, red color represents gassy region, blue color represents empty region, green color represent cluster region and uncolored part is solid region. Bi-direction arrow represents the direction of vibration. Small red arrow and black circular arrow represents the direction of convection.

2, 3 and a gassy region appeared after the empty region in corner 3 as shown in Fig. 6c.

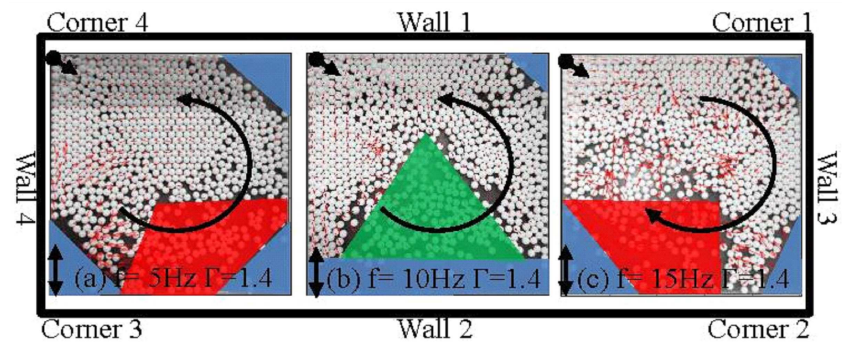

Fig. 6. PIV results: region appeared in 3D state mode, red color represents gassy region, blue color represents empty region, green color represents cluster region and uncolored part is solid region. Bi-direction arrow represents the direction of vibration. Small red arrows and black circular arrow represents the direction of convection.

\subsection{Regions kinetic energy effects on convection mode}

Energy is derived from the vibrated walls 1 and 2 of the container and average kinetic energy of each region affects the mechanics behind the convection motion. Kinetic energy of regions depends on the mass distribution, region size, friction between the particles, and collisions of particles with one another and with the walls of container. The particle moved very quickly in gassy region and forced the system into convection. When force was exerted by gassy region from the opposite sides of the container equally, then no convection was observed, and when the gassy region was at any one side of the system, then the unbalance force exerted by gassy region particles drove the system into convection motion. Area 
covered by the gassy and empty regions increased with dimensionless acceleration. When $\Gamma=1.4$ particles in gassy region moved rapidly and collided with solid region. Consequently, particles moved towards corner 4 of container. All the gaps between the particles in solid region fully vanished and particles jumped from corner 4 of the container and the system transformed to third dimension 3D state. Different modes of convection, due to differences of kinetic energy of regions, were observed in the system. Empty region size affects the densities of all the regions by increasing them. Cluster region did not show any prominent effect on convection motion. Only solid and gassy regions had a dominant effect on the modes of convection. Kinetic energy factor $\mu$ of gassy and solid regions ratio $\mu=k_{g} / k_{s}$ defines the convection motion. We divide convection mode with respect to $\mu$ as shown in Fig. 7. When $(\mu=0.02)$ non convection mode, $(\mu=5.36)$ two rolls convection mode, $(\mu=6.23)$ $3 \mathrm{D}$ state mode, $(\mu=6.85)$ one roll clockwise convection mode, and $(\mu=8.76)$ one roll anticlockwise convection mode were observed.

\section{Conclusions}

Horizontally vibrated monolayer of granular particles at low frequency $f$ and low dimensionless acceleration $\Gamma$ exhibited four different regions: solid region, cluster region, gassy region, and empty regions. Gassy region particles move rapidly and collide with solid region particle, therefore, the density of solid region reaches maximum. Consequently, the system extended to 3D state. Different modes of convection, with respect to kinetic energy factor of gassy and solid regions $\mu=k_{g} / k_{s}$, interpret the modes of convection as $(\mu=0.02)$ non convection mode, $(\mu=5.36)$ two rolls convection mode, $(\mu=6.23)$ $3 \mathrm{D}$ state mode, $(\mu=6.85)$ one roll clockwise convection mode, and $(\mu=8.76)$ one roll anticlockwise convection mode.

\section{Acknowledgments}

National Natural Science Foundation of China (51476009) sponsored this article.

\section{References}

[1] J.S. Olafsen, J.S. Urbach, Phys. Rev. Lett. 81, 4369 (1998).

[2] M.G. Clerc, P. Cordero, J. Dunstan, K. Huff, N. Mujica, D. Risso, G. Varas, Nat. Phys. 4, 249 (2008).

[3] I.S. Aranson, L.S. Tsimring, Rev. Mod. Phys. 78, 641 (2006).

[4] G.H. Ristow, Pattern Formation in Granular Matter, Springer, Berlin 2000.

[5] J.M. Ottino, D.V. Khakhar, Ann. Rev. Fluid Mech. 32, 55 (2000).

[6] T. Mullin, Phys. Rev. Lett. 84, 4741 (2000).

[7] K.C. Chen, C.C. Li, C.H. Lin, G.H. Guo, Phys. Rev. E 79, 021307 (2009).
[8] I. Goldhirsch, G. Zanetti, Phys. Rev. Lett. 70, 1619 (1993).

[9] M.E. Möbius, Xiang Cheng, G.S. Karczmar, S.R. Nagel, H.M. Jaeger, Phys. Rev. Lett. 93, 198001 (2004).

[10] N. Rivas, A.R. Thornton, S. Luding, D. van der Meer, Phys. Rev. E 91, 042202 (2015).

[11] F. Melo, P. Umbanhowar, H.L. Swinney, Phys. Rev. Lett. 72, 172 (1994).

[12] S.T. Thoroddsen, A.Q. Shen, Phys. Fluids 13, 4 (2000).

[13] Royer, J.R., D.J. Evans, L. Oyarte, Q. Guo, E. Kapit, M.E. Möbius, S.R. Waitukaitis, H.M. Jaeger, Nature 459, 1110 (2009).

[14] R. Ramírez, D. Risso, P. Cordero, Phys. Rev. Lett. 85, 1230 (2000).

[15] J.A. Gallas, H.J. Herrmann, S. Sokolowski, Phys. Rev. Lett. 69, 1371 (1992).

[16] J.B. Knight, H.M. Jaeger, S.R. Nagel, Phys. Rev. Lett. 70, 3728 (1993).

[17] Fuweng Zhang, Li Wang, Chuanping Liu, Ping Wu, Shuai Zhan, Phys. Lett. A 378, 1303 (2014).

[18] S. Tennakoon, L. Kondic, R. Behringer, Europhys. Lett. 45, 470 (1999).

[19] G. Metcalfe, S.G.K. Tennakoon, L. Kondic, D.G. Schaeffer, R.P. Behringer, Phys. Rev. E 65, 031302 (2002).

[20] S. Aumaitre, C. Puls, J.N. McElwaine, J.P. Gollub, Phys. Rev. E 75, 061307 (2007).

[21] K. Liffman, G. Metcalfe, P. Cleary, Phys. Rev. Lett. 79, 4574 (1997).

[22] A. Rehman, Ping Wu, Li Li, Shiping Zhang, Li Wang, Acta Phys. Pol. A 130, 1336 (2016).

[23] T. Poschel, D.E. Rosenkranz, J.A. Gallas, Phys. Rev. E 85, 031307 (2012).

[24] A. Raihane, O. Bonnefoy, J.-L. Gelet, J.-M. Chaix, G. Thomas, Powder Technol. 190, 252 (2009).

[25] S.-S. Hsiau, M.-Y. Ou, C.-H. Tai, Adv. Powder Technol. 13, 167 (2002).

[26] M. Medved, Phys. Rev. E 65, 021305 (2002).

[27] S. Nadler, O. Bonnefoy, J.-M. Chaix, G. Thomas, J.L. Gelet, Eur. Phys. J. E 34, 66 (2011).

[28] M. Medved, D. Dawson, H.M. Jaeger, S.R. Nagel, Chaos Interdisc. J. Nonlin. Sci. 9, 691 (1999).

[29] A. Rehman, Ping Wu, S. Shoukat, Li Wang, Int. J. Emerg. Trends Sci. Technol. 04, 6328 (2017).

[30] G.H. Ristow, G. Straßburger, I. Rehberg, Phys. Rev. Lett. 79, 833 (1997).

[31] J.A.C. Gallas, H.J. Herrmann, S. Sokolowski, J. Phys. II (France) 2, 1389 (1992).

[32] C. Salueńa, T. Pöschel, S.E. Esipov, Phys. Rev. E 59, 4422 (1999).

[33] H.M. Jaeger, S.R. Nagel, R.P. Behringer, Rev. Mod. Phys. 68, 1259 (1996).

[34] A. Rehman, Ping Wu, Li Li, Shiping Zhang, Li Wang, Chin. J. Phys. 55, 1713 (2017). 